Cahiers $d u$ MONDE RUSSE

\section{Cahiers du monde russe}

Russie - Empire russe - Union soviétique et États indépendants

$50 / 2-3 \mid 2009$

L'Europe orientale, 1650-1730. Crises, conflits et renouveau

\title{
Renovating RussiaDaniel BEER
}

, Ithaca - Londres : Cornell University Press, 2008, 229 p.

\section{Benjamin Guichard}

\section{(2) OpenEdition}

\section{Journals}

Édition électronique

URL : https://journals.openedition.org/monderusse/9752

DOI : $10.4000 /$ monderusse.9752

ISSN : $1777-5388$

Éditeur

Éditions de l'EHESS

Édition imprimée

Date de publication : 15 septembre 2009

ISBN : 978-2-7132-2260-3

ISSN : $1252-6576$

Référence électronique

Benjamin Guichard, "Renovating RussiaDaniel BEER », Cahiers du monde russe [En ligne], 50/2-3 |

2009, mis en ligne le 14 janvier 2013, consulté le 03 septembre 2022. URL : http://

journals.openedition.org/monderusse/9752 ; DOI : https://doi.org/10.4000/monderusse. 9752

Ce document a été généré automatiquement le 3 septembre 2022

Tous droits réservés 


\title{
Renovating RussiaDaniel BEER
}

, Ithaca - Londres : Cornell University Press, 2008, 229 p.

\author{
Benjamin Guichard
}

\section{Daniel BEER, Renovating Russia, The Human Science and the Fate of Liberal Modernity, 1880-1930, Ithaca - Londres : Cornell University Press, 2008, 229 p.}

Dans le sillage des courants récents de l'historiographie, qui remettent en cause la césure radicale de 1917 dans l'histoire russe, Daniel Beer se penche sur les sources intellectuelles des pratiques politiques bolcheviques, pour montrer qu'elles puisent leur inspiration dans les conceptions scientistes de la réforme sociale, élaborées en dehors des cercles intellectuels marxistes au tournant des $\mathrm{XIX}^{\mathrm{e}}$ et $\mathrm{Xx}^{\mathrm{e}}$ siècles. Il cherche ainsi à retracer les discussions sur la modernisation de la société russe dans le domaine des "sciences humaines", expression qui recouvre les travaux de psychiatres et de criminologues relatifs aux déviances sociales, à l'alcoolisme ou à la violence collective. Le corpus est organisé autour de la discussion des notions de dégénérescence (vyroždenie) et de régénération sociale (ozdorovlenie). L'auteur étudie ainsi les liens qui sont tissés entre l'étude de la criminalité et les analyses de la société pour dégager les stratégies de prophylaxie sociale et de répression qui en découlent. Sa thèse est que les scientifiques russes développent une analyse originale du poids des facteurs héréditaires et environnementaux responsables de la déviance sociale, à l'origine d'une légitimation du recours à la violence et à la coercition. Une action autoritaire de l'État apparaît ainsi comme la seule réponse aux menaces de décomposition sociale et comme la voie unique de dépassement des blocages économiques et politiques du pays.

2 Le premier chapitre présente cette théorie de la dégénérescence. Liant de façon dynamique crime et crise sociale, celle-ci présente les différentes déviances individuelles comme une menace sociale grandissante à l'heure de la transformation des sociétés modernes par l'industrialisation et l'urbanisation. La matrice de ces analyses organicistes est attribuée à l'aliéniste français Bénédict Morel. La réception de ces thèses par les psychiatres et les juristes russes est étudiée en détail et replacée dans le contexte plus 
large de la fortune du darwinisme social. Celui-ci contribue à généraliser l'emploi d'un vocabulaire emprunté à la biologie pour analyser le corps social. Le deuxième chapitre expose les implications de ces théories dans le contexte russe, en défendant l'idée que les tenants de cette biopsychologie sont tous des adversaires de l'ordre social tsariste dont ils réclament la réforme. Les événements de 1905 bouleversent ces analyses, l'intelligentsia étant prise dans le paradoxe du double rejet de l'autocratie et de la condamnation des violences révolutionnaires dont l'impact traumatique fait l'objet d'études cliniques. Le troisième chapitre se penche sur la réception de l'anthropologie criminelle de l'Italien Cesare Lombroso : si l'atavisme défendu par Lombroso est rejeté au profit des facteurs sociaux, les scientifiques russes défendent la thèse de la transmission héréditaire des caractères pathologiques acquis. La prophylaxie du crime devient ainsi un enjeu social de grande ampleur et conditionne la possibilité de réforme politique et sociale du pays. Ces analyses légitiment le recours à la coercition, l'enfermement préventif et la rééducation par le travail. Le chapitre suivant est consacré aux échos des thèses de Gabriel Tarde sur l'imitation ou de Gustave Le Bon sur la psychologie des foules. En Russie, ces analyses nourrissent la crise de confiance de l'intelligentsia à l'égard des Lumières et dans la possibilité d'une éducation rationnelle du peuple au régime des droits, au profit de formes autoritaires d'ingénierie sociale, seules capables de transformer les masses. Les quarante pages du dernier chapitre cherchent à montrer comment ces thèses, conjuguées au discours marxiste de la révolution, nourrissent les pratiques politiques des premières années du régime soviétique: les débats sur la psychologie sociale se poursuivent sous la NEP et le projet de régénération sociale élaboré au tournant du siècle est mis en œuvre lors des répressions staliniennes à travers un nouveau vocabulaire politique, la prophylaxie de la transmission des caractères acquis passant du terrain de la biologie à celui de la lutte des classes. Daniel Beer peut ainsi conclure que les "praticiens libéraux dans le domaine des sciences humaines en Russie » ont fourni un outillage théorique de la «coercition modernisatrice » mise en œuvre par l'État soviétique et relayée par l'idéologie révolutionnaire (p. 205-206).

Cet effort pour revisiter l'histoire intellectuelle du libéralisme russe et de l'ingénierie sociale est séduisant ; il fait écho aux entreprises d'historiens comme Yanni Kotsonis et Peter Holquist, voire aux travaux sur l'Allemagne hitlérienne de Zygmunt Bauman (qui n'est cité qu'en toute fin d'ouvrage, p. 202). Les modalités de la démonstration et les outils méthodologiques utilisés par l'auteur suscitent cependant plusieurs réserves.

4 L'objet de l'étude est imparfaitement délimité. La notion de "sciences humaines » est utilisée sans être discutée. Elle renvoie pour l'auteur aux champs de la psychiatrie, de la criminologie, de la psychologie, auxquels il adjoint, épisodiquement, le droit notamment en introduction et en conclusion, en se penchant sur la figure de Bogdan A. Kistjakovskij. Les sciences sociales à proprement parler, l'anthropologie ou la sociologie, développée à cette période par une figure comme Maksim M. Kovalevskij, sont ignorées. De même, l'auteur présente tous les acteurs qu'il cite comme des "libéraux ", définis comme des " partisans d'une réforme graduelle et contrôlée de l'ordre social, politique et économique ", répondant aux principes de l'empirisme et de la rationalité (p. 2). Il faut donc entendre par là qu'il s'agit de tous ceux qui ne se réclament pas du marxisme et qui ne sont pas des responsables politiques du régime tsariste. Avec Vladimir M. Behterev, les figures les plus citées dans l'ouvrage sont celles des psychiatres Pavel I. Kovalevskij et Ivan A. Sikorskij. Ils sont pourtant proches des milieux monarchistes et nationalistes, le dernier intervenant même au procès Bejlis comme expert cité par le gouvernement ${ }^{6}$. Les 
rapprocher des juristes Vladimir Nabokov ou Pavel Ljublinskij mériterait pour le moins d'être plus amplement discuté.

5 Cette imprécision repose sur un problème de méthode. L'histoire des idées que propose Daniel Beer fait dialoguer des textes. La sélection de ce vaste corpus n'est pas explicitée, mais repose probablement sur l'occurrence des notions de dégénérescence et de régénération sociale. Le statut de ces différentes sources imprimées n'est pas exploité et la démonstration mêle l'extrait d'une revue médicale spécialisée avec l'article d'une revue de vulgarisation destinée au grand public comme Vestnik Znanija. La biographie des auteurs, leur parcours professionnel et intellectuel, leur sociologie et leurs affiliations politiques, ne sont jamais étudiés. Daniel Beer préfère reconstituer le «consensus professionnel » de l'époque - expression employée à plusieurs reprises -, plutôt que de restituer les lignes de fracture qui traversent ces débats. Certes, il s'agit pour l'auteur de s'intéresser à l'emprise sociale et culturelle de ces thèses ; mais peut-on faire l'économie d'une étude systématique du développement institutionnel et social des disciplines psychiatriques et criminologiques qu'il étudie? Il aurait également été précieux de faire dialoguer ces thèses avec les pratiques pénales, judiciaires et thérapeutiques développées par ces mêmes figures, de retracer la fortune de ces analyses dans les pratiques institutionnelles tsaristes et soviétiques. Faute d'un travail d'archives, les analyses de l'auteur ne valent pas véritablement démonstration.

Enfin, si la question de la généalogie intellectuelle des pratiques bolcheviques est un sujet à part entière, il est dommage que l'analyse du corpus soit limitée à cette seule perspective. Dans ces débats de médecins et de juristes russes sur les causes du crime et sa prophylaxie, l'eugénisme n'est discuté que rapidement (p. 123-124) et l'on s'étonne de ne trouver aucune mention des débats sur la question juive et les nationalités de l'Empire.

7 Daniel Beer a ainsi mis à jour et organisé un corpus passionnant, mais l'analyse et la mise en contexte de ces sources laissent le lecteur sur sa faim.

\section{NOTES}

6. Laura Engelstein, The Keys to Happiness: Sex and the Search for Modernity in Fin-de-Siècle Russia, Ithaca-Londres : Cornell University Press, 1992, p. 326. 